\title{
Labyrinthe
}

$22 \mid 2005$ (3)

La Biopolitique (d')après Michel Foucault

\section{La population contre le peuple}

L'agonistique masquée des cours de Foucault au Collège de France, 1977-1979

Déborah Cohen

\section{OpenEdition}

1 Journals

Édition électronique

URL : http://journals.openedition.org/labyrinthe/1035

DOI : $10.4000 /$ labyrinthe. 1035

ISSN : 1950-6031

Éditeur

Hermann

Édition imprimée

Date de publication : 1 novembre 2005

Pagination : 67-76

Référence électronique

Déborah Cohen, "La population contre le peuple », Labyrinthe [En ligne], 22 | 2005 (3), mis en ligne le 22 juillet 2008, consulté le 07 mai 2019. URL : http://journals.openedition.org/labyrinthe/1035 ; DOI : 10.4000/labyrinthe. 1035 


\title{
LA POPULATION \\ CONTRE LE PEUPLE \\ L'agonistique masquée des cours de Foucault au Collège de France, 1977-1979
}

\author{
Deborah COHEN \\ cohen.deborah@free.fr
}

Foucault n'aimait guère l'histoire des idées telle qu'on pouvait majoritairement la pratiquer à son époque. De celle qui faisait se succéder les théories comme autant de tentatives de plus en plus réussies, mais toujours incertaines, pour accéder au concept contemporain achevé, il ne pouvait que refuser la façon d'hypostasier les concepts, la recherche d'un universel présent dans la pensée passée. À celle qui isolait la spécificité d'une école ou d'un individu et s'intéressait aux volontés de l'auteur, il opposait la nécessité de «définir une méthode d'analyse qui soit pure de tout anthropologisme ${ }^{1} »$. Et surtout, il ne fallait pas, comme le faisait l'histoire marxiste des idées, rechercher la cause de constitution de certains concepts, relier à une idée un contexte (économique notamment) qui serait la raison de son émergence. L'histoire de l'humanité n'était ni celle d'une raison reflétée dans des consciences, ni un matérialisme dialectique.

Or, une nouvelle histoire des idées, essentiellement anglo-saxonne et d'inspiration wittgensteinienne, a permis que soit déplacée la question des causes d'un événement discursif. L'école de Cambridge se met en place vers la fin des années 1960 autour de John Pocock puis de Quentin Skinner. Très mal connus en France jusque récemment, leurs travaux n'étaient cependant pas étrangers à Foucault ${ }^{2}$. Refusant, comme Foucault, et le récit continu d'un progrès de l'idée vraie vers sa plénitude et l'explication purement contextuelle, ces auteurs s'intéressent au sens de

\footnotetext{
1. Foucault, L'Archéologie du savoir, Paris, Gallimard-NRF, 1969, p. 26.

2. Voir par exemple la mention (certes non développée) qu'il en fait en 1967 dans un entretien avec Raymond Bellour: « Sur les façons d'écrire l'histoire », repris dans Dits et Écrits, 1954-1988, vol.1, Gallimard-NRF, 1994, p. 585 et 587.
} 
l'événement linguistique lui-même, dont connaître la cause ne nous dit rien. Ils cherchent à saisir l'intention qu'avait l'auteur en prononçant ou écrivant une proposition. Parler, écrire, c'est faire, c'est agir en fonction d'un but. Pour saisir le sens de cet acte de parole, il s'agit donc de comprendre de quelle manière il pouvait être reçu. L'auteur se sert des moyens linguistiques qui sont à sa disposition, et c'est donc sur fond de l'ensemble des possibles, des propositions conformes, des autres énoncés qui peuvent lui être liés, que viendront prendre sens les propositions d'un texte.

Sans recourir à des explications causales, ce que réintroduisent des analyses de ce type, c'est la possibilité d'une agonistique, d'un heurt entre les propositions. Lorsque surgissent, parmi les termes anciens du débat, des formulations neuves, on peut y lire une intention de l'auteur (qui n'est pas forcément à entendre comme provocation consciente) dont les enjeux sont souvent politiques. Il m'a semblé que les deux cours Sécurité, territoire, population et Naissance de la biopolitique, en délimitant justement l'espace du pensable et du dicible en un temps donné, fournissaient (sans jamais la faire) tous les éléments d'une histoire des luttes d'idées qui ont couru à la fin du XVIII ${ }^{\mathrm{e}}$ siècle, entre une voie que Foucault dit juridico-déductive, voie de la Révolution française ou voie rousseauiste ( $N B$, p. 44$)$, et celle du libéralisme naissant. C'est de ces affrontements, qui courent sous l'histoire quelque peu pacifiée que nous présente Foucault, que je me soucierai ici.

\section{Pouvoir-savoir, conduites et savoirs d'opposition}

On retrouve dans les deux cours de la fin des années 1970 la notion de pouvoir-savoir déjà présente dans Surveiller et Punir ou dans La volonté de savoir. Ce sont certaines structures de pouvoir, un ensemble d'institutions notamment, qui suscitent les formes de savoir dont elles se nourrissent. Ainsi la statistique est-elle certes ce qui, au XVIIII siècle, rend la police possible, mais aussi la forme de savoir qu'a rendu nécessaire - et donc pensable - cette police (STP, p. 323). L'économie politique apparaît alors comme la forme de savoir nécessairement associée à cette forme de pouvoir qu'est la gouvernementalité. Pourtant, si le pouvoir se définit d'être un certain savoir, à l'origine de cette économie politique 


\section{La population contre le peuple}

les physiocrates ont, notamment à l'image d'un courant d'opposition juridique, pensé des formes de rupture possible de cette union. Ce faisant, ils ont participé (d'une manière que Foucault met peut-être trop de côté) d'un moment d'affirmation d'une extériorité posée face à la raison d'État.

Fin XVI'-début XVII ${ }^{\mathrm{e}}$ siècle, nous dit Foucault, s'impose le modèle politique de la raison d'État, où l'activité gouvernementale ne prolonge ni souveraineté divine ni lois de la nature. Gouverner est un art de l'artificiel, qui ne se rapporte à rien d'autre qu'à la nécessité de l'État lui-même. La loi politique, pour se construire, se cale alors sur un certain nombre de données du monde, analysées, organisées de manière à former un nouveau savoir stable et maîtrisable par le pouvoir. Ainsi dépourvues de leur références naturelles et divines, ces données se trouvent en somme réduites au statut de choses au service de l'État. Plutôt que sur un ensemble de sujets de volonté, c'est sur des corps disciplinables que porte le pouvoir désormais. De la vie de ces corps il est possible de donner une description phénoménologique claire. Ainsi les révoltes sont-elles pensables, chez Bacon ou chez Machiavel, comme des phénomènes quasi physiques. Sous leur description tranquille, le peuple en émeute devient l'objet ordinaire d'une « physique » nouvelle du pouvoir (STP, p. 273 sqq.).

Objectivés par le savoir d'un État disciplinaire, les sujets du souverain deviennent des corps - dociles ou rebelles. Plus qu'à des sujets, c'est donc à une population que l'on aurait affaire. Pourtant, Foucault hésite. Implicitement définie par la nature des objets auxquels s'applique la raison d'État, la population n'existe pour ainsi dire pas encore, car elle n'est pas entrée dans son moment réflexif (STP, p. 283). Il ne s'agit pas simplement pour lui de préserver la spécificité du moment disciplinaire analysé dans Surveiller et Punir, mais de marquer que la dispersion des corps singuliers n'est réellement formée en population que par l'intermédiaire de l'institution policière qui « gère » les mouvements à l'échelle du royaume, qu'elle est contemporaine d'un moment où l'on veille non pas seulement à la discipline mais à la sécurité des organismes. Cette entrée dans un «prisme réflexif » n'en reste pas moins assez floue; la détermination de son moment et de ses modalités n'intéresse pas du tout Foucault.

On fera ici l'hypothèse, en partant largement des textes mêmes de Foucault, que ce moment surgit non pas dans la seule continuité des agencements institutionnels nouveaux, mais comme explicitation nécessaire, 
construite contre ce qui, dans la pratique ou les discours, résistait à cette nouvelle forme de structuration du réel.

Il faut pour cela mettre l'accent sur ce qui, de manière surprenante, se trouve quelque peu effacé du récit foucaldien. Ce n'est guère jouer le rôle de l'historien érudit que de rappeler ici ce que chacun sait: les XVII et XVIII ${ }^{\mathrm{e}}$ siècles sont temps de rébellion. Quoique passée au crible d'un savoir-pouvoir qui l'objective, qui de sujets de volonté tendrait à ne laisser subsister que des objets à contrôler, la population n'en demeure pas moins peuple. Face au pouvoir se dressent ce que Foucault appelle des contre-conduites, qui ne prennent d'ailleurs pas toujours la forme d'un simple retournement des normes qui, sous forme négative, accomplirait en fait la même logique. En effet, les émeutes modernes ne sont pas de pures émeutes frumentaires, ne sont pas simplement le surgissement incontrôlé d'une population insatisfaite, mais elles revêtent des aspects proprement politiques. C'est que la raison d'État a constitué « le public comme sujet-objet d'un savoir: sujet d'un savoir qui est opinion » (STP, p. 281). Aussi réglés que soient les comportements séditieux, aussi connues que soient les réponses que l'on cherche à leur donner par l'intermédiaire d'une police multiforme, ils n'en demeurent pas moins imprévisibles. Il y a bien un reste, celui de ceux qui s'insurgent. Ces comportements qui ne rentrent pas dans ce « sujet-objet collectif » dont s'occupe la raison d'État, et « vont dérégler le système », c'est ce que, tel que Foucault le cite, le physiocrate Abeille, dans sa Lettre d'un négociant de 1763, appelle le peuple (STP, p. 45).

Tandis que se manifestent ainsi concrètement les « résidus » d'une objectivation incomplète, la raison d'État suscite les formes des discours qui s'opposent à elle. Mais sur cela, Foucault ne s'attarde pas plus que sur les grondements de l'opinion publique. Tout juste évoque-t-il, de manière englobante et sans préciser, l'existence, face à la raison d'État, d'un courant de critique juridique (STP, p. 355). Pourtant, ce sont bien les juristes qui, rompant avec l'idée d'un pouvoir-savoir sans extériorité, vont inventer un vis-à-vis à la raison d'État ${ }^{3}$. Dans le cadre d'un pouvoir dont les lois ne sont plus d'origine divine, une forme de critique à partir de l'extériorité va pouvoir se faire jour. Mais de toutes les figures qui, face au pouvoir, construisent la légitimité d'une volonté autre (qu'elle

3. Foucault évoque, en passant, « ces lois fondamentales du royaume que les juristes vont objecter à la raison d'État » $(N B$, p. 10$)$. 


\section{La population contre le peuple}

soit populaire ou non), il n'est jamais ou presque jamais question dans les deux cours. De Hobbes, de Rousseau, de Montesquieu, point.

Rappeler brièvement la vitalité de ces contre-conduites et de cette tradition juridico-légale, qui met en question la légitimité du pouvoir à confondre et à maîtriser la totalité du savoir et du pouvoir, permet de voir sur quel fond vont venir s'inscrire les pensées libérales. La réaction d'Abeille, singularisant un peuple rebelle, nous montre que c'est sur fond d'une mise en visibilité du peuple concret que va pouvoir s'énoncer le portrait de son contraire, la population. De même, il peut ne pas être inutile de garder en tête que ce par quoi sont travaillés les apprentis-libéraux du XVIII ${ }^{\mathrm{e}}$ siècle, c'est avant tout par un débat juridicolégal offrant un terreau de légitimité à des formes d'opposition extérieures à l'État.

\section{Les physiocrates, du contre-pouvoir populaire au silence de la population}

À lire la fresque de Foucault, les physiocrates sont sortis pour ainsi dire tout armés de la raison d'État et des dispositifs de sécurité mis en place. Il ne peut s'agir pour lui de trouver «la cause de la constitution du marché comme instance de véridiction » $(N B$, p. 35$)$, mais de déterminer le champ des possibles sur lequel ont pu s'inscrire les discours. Pourtant, dans la mesure où Foucault ne nous dit rien des occasions avortées, du point exact d'inflexion où le possible sombre dans l'impossible, les objets qu'il décrit tendent à apparaître (quoiqu'il s'en défende) comme absolument nécessaires.

Or, les physiocrates se sont justement situés sur une dangereuse ligne de partage des eaux : d'un côté, les théories juridico-légales dont ils assument bien des prémisses; de l'autre, une plongée vers la société économique. On peut penser que la signification de ce qui est advenu ne peut bien se comprendre que dans une confrontation avec son autre abandonné; la signification d'un texte s'enracine dans ce qu'il a refusé d'être et que chacun attendait. Le concept de population peut être mieux compris si on le replace dans la continuité déviante de son autre, le concept de peuple. C'est dans le dialogue (et peut-être vaudrait-il mieux dire le combat) avec celui-ci que se construit celui-là. Ce dialogue est tacite, le concept politique de peuple faisant partie du fond commun de 
référence à partir duquel pensent les physiocrates. Il est aussi explicite, les ouvrages s'enchaînant et se répondant en une polémique, dont Foucault tait jusqu'au nom des principaux protagonistes, notamment celui de Mably, celui de Galiani, celui de Diderot.

Un certain nombre de thèmes sont communs aux théories juridicolégales et à l'économie politique naissante. Tout d'abord, comme sous le régime de la raison d'État le souverain n'est plus l'expression d'une volonté divine, son pouvoir doit connaître des limites, et il peut donc y avoir abus de l'autorité. C'est une figure extérieure au pouvoir qui peut prendre la mesure de cet abus. La nation est une de ces figures possibles, détentrice d'une vérité sur le pouvoir et ses limites. Or, les physiocrates non seulement reconnaissent une place à cette figure d'extériorité que peut être l'opinion de la nation éclairée, envisagent la possibilité d'une représentation locale des principaux agents économiques, mais explorent les voies d'une possible vérité (sinon politique, du moins morale) au sein des couches populaires. À ces individus responsables il n'est donc pas possible a priori d'imposer comme seul principe celui de l'obéissance passive. Les hommes entrant en société conservent absolument tous leurs droits d'ordre juridique et moral; l'intérêt commun est inséparable de la justice 4 . Toute entrave qui leur est faite constitue alors un « abus de l'autorité ${ }^{»}$. Dans la dédicace de son traité De l'ordre social, Le Trosne félicite donc le Margrave de Bade d'avoir mis « ses sujets en état de juger par eux-mêmes des principes » de son administration ${ }^{6}$.

La confiance accordée à l'opinion apparaît clairement chez les physiocrates à l'occasion du débat sur l'éducation du peuple. Mably (que l'on peut considérer comme un bon représentant de cette voie juridicodéductive ou révolutionnaire que Foucault pose comme radicalement hétérogène à celle des tenants du marché) tente de s'appuyer sur cet élément: «On veut que le peuple soit ignorant; mais remarquez, je vous prie, qu'on n'a cette fantaisie que dans les pays où l'on craint la liberté [...]. Je crois en effet que si les citoyens sont bien sots, bien stupides, bien ignorants, ils vivront dans le repos: mais quel cas vous et moi devons-nous faire de ce repos? Il ressemble à cet engourdissement qui

\footnotetext{
4. $\rightarrow$ Voir l'article de L. Loiseau. $\nleftarrow$

5. Le Trosne, De l'ordre social. Ouvrage suivi d'un traité élémentaire sur la Valeur, l'Argent, la Circulation, l'Industrie et le Commerce intérieur et extérieur, à Paris chez les frères Debure, 1777, p. 41. 6. Ibid., p. IX.
} 


\section{La population contre le peuple}

lie les facultés d'un paralitique $[\mathrm{sic}]^{7}$.» De tels propos ne seraient pas désavoués par Le Trosne qui assure en effet: «Dans les gouvernements déréglés l'instruction du peuple est absolument négligée: on va même jusqu'à prétendre qu'elle est dangereuse, et qu'elle rend le peuple moins docile, plus impatient et plus disposé à la révolte. Oui sans doute il est à propos de le dévouer à l'ignorance, lorsqu'on veut le gouverner à discrétion, comme un vil troupeau ${ }^{8}$.»

Il y a donc bien un fond commun aux économistes et au courant juridico-légal, et ce fond concerne la nécessaire liberté d'une opinion, une possible confrontation entre le pouvoir et ceux qui lui sont extérieurs. Le peuple, comme sujet politique, existe bien dans la pensée physiocratique. Mais c'est justement parce que ce peuple existe dans leur horizon réflexif qu'on ne peut faire l'économie d'essayer de comprendre dans quel rapport se tient avec lui ce «personnage politique absolument nouveau » (STP, p. 61) qu'est la population. C'est précisément à partir de ce point même que va s'insinuer la rupture.

S'il n'y a plus de lois naturelles ou divines qui, maîtrisées par le souverain, puissent servir de clé assurée pour une bonne politique, nul ne peut plus être sûr de ce qu'est la politique. Le mot lui-même fait partie des abstractions que remet en cause une méthode sensualiste ne déduisant les idées que d'une origine sensorielle. On trouve chez les physiocrates comme chez les tenants d'un courant juridico-légal une même tentation nominaliste sur ce point. Vous verrez, écrit Le Trosne, «les hommes, uniquement d'accord sur les mots, convenir que le bonheur des citoyens est le but de l'association, et se partager à l'infini sur les moyens de le procurer ${ }^{9} \gg$.

L'objet du débat a donc été mal défini, on a réfléchi à partir de mots creux, débridant la métaphysique. Ce faisant, les physiocrates se trouvent d'accord, du moins en apparence, avec tous ceux qui, comme Mably, pensent qu' " il n'y a rien en morale ni en politique sur quoi les gens qui ont l'esprit le plus exercé et le plus de lumières ne se trouvent partagés ${ }^{10}{ }$. D'accord sur le constat préalable, Le Trosne et Mably se divisent sur les

7. Gabriel Bonnot de Mably, Des droits et des devoirs du citoyen, Paris, éd. critique de Jean-Louis Lecercle, 1972, p. 57.

8. Le Trosne, op. cit., p. 270-271.

9. Ibid., p. 56.

10. Dans Doutes proposés aux philosophes économistes sur l'ordre naturel et essentiel des sociétés politiques, À La Haye et se trouve à Paris, chez Nyon et la Veuve Durand, 1768, p. 56. 
conséquences qu'il faut en tirer. Pour ce dernier, si l'« on n'attache pas les mêmes idées aux mêmes mots qu'on employe » en politique, c'est que la chose est par définition impossible, «les Politiques et les Moralistes méditant sur des questions très compliquées ${ }^{11} \gg$. La politique ne peut être objet d'un savoir maîtrisé, ni de la part du souverain ni de celle de cette extériorité multiforme dressée face à lui. Ne reste plus alors qu'un domaine de confrontations des pures volontés individuelles. Dans ce cadre, l'État est sans cesse soumis au danger du désaccord et de l'émeute. C'est à ce risque d'une politique qui, de savoir du souverain deviendrait objet des volontés du peuple, que réagissent les physiocrates. L'économie politique telle qu'elle se construit à la fin du XVIII siècle peut alors apparaître non plus seulement comme la conséquence nécessaire d'un faisceau de réorganisations institutionnelles dans les pratiques de pouvoir, mais aussi comme une création conceptuelle au combat contre une définition du politique comme univers des valeurs et des volontés.

Deux voies pour cela: redéfinir les sujets, couper court au risque de la volonté en en faisant des objets rationnels analysables; d'autre part et corrélativement, prendre le risque à la racine, redéfinir le concept de politique, trouver les lois sur lesquelles on puisse se mettre d'accord.

Comment donc redéfinir les sujets pour éliminer le risque d'une révolution légitime, et cela, sans pour autant entrer dans le schéma disciplinaire d'une « obéissance totale et en quelque sorte passive des individus à leur souverain » (STP, p. 67), dont on a vu qu'il était inacceptable pour les physiocrates? En se branchant, nous dit Foucault, non plus « sur l'axe du rapport souverain-sujet », mais « sur des processus que les physiocrates disaient physiques » (idem). Les cours de Foucault sont absolument précieux pour cerner ce passage d'une politique pensée en termes de volontés dans un rapport souverain-sujet à une économie pensée en termes d'intérêts sur un axe gouvernement-objet. Il me semble juste que l'aspect politique du passage se trouve par lui gommé. Il s'agit de déplacer un problème formulé en des termes devenus trop dangereux. À un lien politique volontaire mais incertain il s'agit de substituer un lien économique. L'objet économique apparaît comme la réponse, car il se trouve lié aux autres d'une manière qui concilie douceur et assurance ${ }^{12}$.

11. Ibid., p. 57.

12. «L'obéissance est due au souverain: pourquoi vouloir l'obtenir par la contrainte, tandis qu'il est si aisé de la rendre douce et facile? [...] C'est par l'intérêt, c'est par leur bonheur qu'il doit attacher ses 


\section{La population contre le peuple}

L'invention de la population est réponse au risque du peuple. Si tout un faisceau de dispositifs institutionnels a rendu possible cette émergence, sa formulation claire ne s'en est pas moins faite dans un champ discursif structuré comme une arène.

Les sujets politiques vivent dans un monde de l'incertain - incertitude qui tient aux passions humaines, aux hasards de l'histoire. C'est parce que la politique est un univers d'ignorance généralisée que toutes les opinions se valent et qu'il n'est pas d'expertise possible. C'est aussi par rapport à ces positions que les physiocrates doivent définir leur place. C'est pour repousser « le sable mouvant et sans consistance de l'opinion arbitraire et versatile ${ }^{13} \gg$ qu'il leur faut définir la politique comme relevant de l'ordre des vérités géométriques. Lorsque l'ordre social et ses règles seront connues (d'où l'utilité d'une éducation, y compris populaire), son «pouvoir irrésistible » lui soumettra « tous les esprits et tous les cœurs, amènera sans effort et sans violence la plus entière et la plus heureuse révolution dans la législation, dans les mœurs, dans le gouvernement, dans la politique ${ }^{14} »$. Ainsi se trouve repoussé, pour les physiocrates, le risque d'une extériorité posée face au pouvoir, sans pour autant que l'on ait à renoncer à la nécessité de sortir d'un rapport d'obéissance passive à l'interdit de la loi. Si, pour les physiocrates, l'évidence économique est ce qui limite le pouvoir, c'est qu'une limitation par la liberté des individus est trop cher payée.

En conclusion, pour Foucault, «le monde politico-juridique et le monde économique apparaissent, dès le XVIII ${ }^{\mathrm{e}}$ siècle, comme des mondes hétérogènes et incompatibles» ( $N B$, p. 286). Incompatibles, oui sans doute, hétérogènes, peut-être pas. L'homo juridis et l'homo æeconomicus coexistent et bataillent au XVIII ${ }^{\mathrm{e}}$ siècle, le second étant explicitement construit pour prendre la place du premier. Mais il n'y a antagonisme qu'au sens d'une agonistique, d'un combat sur un terrain commun. Il s'agit d'une même tradition de pensée (celle qui affirme la nécessité d'une extériorité demeurant face au souverain) qui s'est ensuite divisée dans la peur et la polémique. Foucault refuse explicitement ce genre

\footnotetext{
sujets à son administration, et il a pour y parvenir des moyens bien différens de la force ». Le Trosne, op. cit., p. 269.

13. Le Trosne, op. cit., p. 56.

14. Ibid., p. 10.
} 
d'analyse qui suit une série et ses divisions, il préfère penser des séries hétérogènes, séparées, multiples. Néanmoins, ce sont les éléments mêmes qu'il met en avant qui permettent ensuite de reconstituer l'arrièreplan sur lequel se détache le heurt des positions. Gommant apparemment les affrontements pratiques ou doctrinaires, ce n'est pourtant pas à une vision pacifiée de l'histoire que conduit Foucault. Pensant le sens des émergences il laisse à son lecteur le soin d'expliciter celui des abandons et des refus qui n'en sont pas moins significatifs. Délimitant l'espace du pensable à un moment donné, il nous confie la tâche d'y entendre nousmêmes « le grondement de la bataille ${ }^{15} »-$ ce qui est peut-être le contraire d'une pacification politique.

15. C'est sur ces mots que s'achève Surveiller et Punir. Naissance de la prison, Paris, Gallimard, 1975, p. 360 . 